\section{Aspectos gerais da interdição em psiquiatria}

\section{Sr. Editor,}

Alguns transtornos psiquiátricos dentro do contexto da psicopatologia forense são fenômenos que em sua maioria determinam a necessidade de uma ação cível, bem como a indicação da realização de uma perícia psiquiátrica. ${ }^{1-3,5}$

O termo ação cível se enquadra no processo da "Capacidade Cível" em que se permite a uma pessoa adquirir direitos e contrair obrigações por conta própria, por si mesma, sem a necessidade de um representante legal. Para a ocorrência de uma ação cível de interdição, faz-se necessário que o indivíduo perca a capacidade de gerir seus bens e sua própria pessoa. Esta situação judicial apresenta-se como a mais freqüente nas perícias psiquiátricas, que incidem freqüentemente na incapacidade total e definitiva, a qual se configura pela perda da autodeterminação da pessoa. ${ }^{3}$

$\mathrm{Na}$ literatura, os quadros psicopatológicos de maior frequiência nas perícias psiquiátricas são as doenças degenerativas do Sistema Nervoso Central (Alzheimer, Parkinson, Demências por Vasculopatias), oligofrenias, psicoses e transtornos mentais agudos (Acidente Vascular Cerebral, Traumatismos Crânio-encefálicos e Neoplasias). ${ }^{4}$

Estudando-se um total de 41 perícias psiquiátricas realizadas entre setembro e outubro de 2001, na grande Campinas, SP (solicitadas tanto pela Justiça Gratuita quanto por particulares), observou-se uma prevalência de casos de retardo mental $(\mathrm{n}=26)$. Estes dados numa análise inicial diferem de outros da literatura. ${ }^{4}$ Os demais quadros psiquiátricos observados foram Demência $(n=9)$, Psicoses Crônicas $(n=7)$ e Epilepsia $(n=1)$. Outro fator importante observado neste levantamento refere-se ao fato de que não houve uma relação direta entre o motivo da ação e a patologia associada. Mesmo em quadros demenciais, os motivos da ação direcionavam apenas para a aquisição do benefício.

Nas perícias movidas através da Justiça Gratuita $(\mathrm{JG}=39)$, as motivações foram, em sua maioria $(n=36)$, com intenção do recebimento dos benefícios previdenciários, divisão de bens $(n=2)$ e gerenciamento dos atos da vida civil $(n=1)$.

Em relação à idade, observou-se que a faixa etária de maior prevalência situava-se entre 20 e 30 anos num total de 31 ações. Para as ações particulares, os dois casos relacionaram-se com o gerenciamento de bens e a idade foi acima dos 60 anos.

A necessidade da perícia psiquiátrica nos casos de ações para uma possível interdição apresenta-se hoje freqüente na realidade brasileira. Este fato solicita deste profissional, cada vez mais, uma especificidade para diagnóstico diferencial, cuja conduta seja adequada a cada caso.

Os diagnósticos aqui levantados não são concordantes com os encontrados na literatura, visto que, nesta amostra, predominaram os casos de retardo mental. ${ }^{4}$ Além do que, a demanda para a realização de perícia psiquiátrica foi mais expressiva na Justiça Gratuita, possivelmente influenciada pelo conteúdo da Lei 6742/93 do Instituto Nacional de Seguridade Social (INSS), que garante aos familiares uma curatela definitiva.

Mediante o exposto, levanta-se a seguinte hipótese: além das diferenças de diagnósticos encontradas, haveria também um perfil psicopatológico conforme a procedência da solicitação que motiva a ação? A resposta a este questionamento, sem dúvida, requer uma investigação criteriosa e com maior abrangência de casos para o seu possível esclarecimento.

\section{Eduardo Henrique Teixeira Sérgio Paulo Rigonatti}

Núcleo Forense do Instituto de Psiquiatria do Hospital das Clínicas da Universidade de São Paulo (NUFOR-Ipq)

Antonio de Pádua Serafim Conselho Científico do NUFOR-Ipq

\section{Referências}

1. Kaplan HI, Sadock BJ. Tratado de psiquiatria. Porto Alegre: Artmed; 1993

2. Rosner R. Principles and practice of forensic psychiatry. USA: Chapman \& Hall; 1994.

3. Oliveira J. Código do processo civil. São Paulo: Editora Saraiva; 1995.

4. Laks J, Rozenthal M, Engelhardt E. Neuropsicologia e psiquiatria legal. Rev Bras Neuro 1996:32(6):217-20.

5. Rigonatti SP, Serafim AP, Barros EL. Temas em psiquiatria forense e psicologia jurídica. São Paulo: Editora Vetor; 2003.

\section{Comentário sobre as diversas formas de calcular confiabilidade entre entrevistadores}

\section{Sr. Editor,}

O artigo "Concordância entre observadores da entrevista semi-estruturada para diagnóstico em psiquiatria da infância, versão epidemiológica (KSADS-E)" contribui enormemente para a consolidação do uso do citado instrumento na nossa prática de pesquisa. Gostaríamos, entretanto, de fazer algumas considerações sobre o método utilizado na análise de confiabilidade entre os entrevistadores que participaram do estudo.

Os autores não especificaram na metodologia do estudo se os diagnósticos foram computados de forma dicotômica ou ordinal. Isto é, não sabemos se os diagnósticos foram considerados apenas como ausentes ou presentes ou, alternativamente, se foi utilizada a categorização própria do KSADS, que admite os diagnósticos clínicos, subclínicos e ausentes. Os autores utilizaram para análise da confiabilidade o coeficiente Kappa, que é apropriado para analisar a concordância entre dois observadores, tanto para respostas dicotômicas como ordinais (Kappa ponderado - Agresti, 1990). ${ }^{1}$ Uma vez que os autores tinham quatro observadores, a concordância foi calculada por meio da média entre os coeficientes Kappa calculados para todos os pares possíveis de observadores.

Como alternativa a esse método, sugerimos o coeficiente de correlação intraclasse (ICC) (Rosner, 2000). ${ }^{2}$ Este método foi desenvolvido originalmente para avaliar a confiabilidade entre dois ou mais observadores que utilizam variáveis com distribui- 
ção normal. Entretanto, vários autores, como por exemplo, Elston (1977), ${ }^{3}$ sugerem também sua utilização para variáveis dicotômicas. Uma aplicação pode ser encontrada em Tsuang et $\mathrm{al}^{4}$ (1995). Além disso, Ridout et $\mathrm{al}^{5}$ (1999) discutem vários estimadores do ICC propostos para dados dicotômicos, sendo alguns deles exatamente iguais aos aplicados a variáveis contínuas.

Assim, os autores poderiam ter utilizado o ICC, independentemente da suposição de distribuição das variáveis, ou seja, os diagnósticos na infância e não teriam necessitado adaptar o Kappa por meio do cálculo das médias dos pares de observadores, um método engenhoso, mas desnecessário.

Mariana Curi e Ana Gabriela Hounie Instituto de Psiquiatria da Faculdade de Medicina da Universidade de São Paulo

\section{Referências}

1. Agresti A. Categorical data analysis. New York: John Wiley \& Sons; 1990. p. 366-70.

2. Rosner B. Fundamentals of biostatistics. California: Duxbury; 2000. p. 562-7.

3. Elston RC. Response to query: estimating "heritability" of a dichotomous trait. Biometrics 1977;33:232-3.

4. Tsuang MT, Tohen M, Zahner GEP. Textbook in psychiatric epidemiology. New York: John Wiley \& Sons; 1995. p. 213-25.

5. Ridout MS, Demétrio CGB, Firth D. Estimating intraclass correlation for binary data. Biometrics 1999;55:137-48.

\section{Guilherme Polanczyk et al responde:}

É com satisfação que recebemos o interesse das colegas Ana Gabriela Hounie e Mariana Curi pelo nosso artigo "Interrater Agreement for the Schedule for Affective Disorders and Schizophrenia, Epidemiological Version for School-Age Children (K-SADS-E)", ${ }_{1}$ recentemente publicado nesta revista.

$\mathrm{Na}$ sua cuidadosa leitura do estudo, elas identificam duas questões que merecem discussão:

a) especificação da forma de apresentação dos diagnósticos (dicotômica ou ordinal). Embora a versão epidemiológica do KSADS, utilizada nesse estudo, permita três codificações (diagnóstico ausente, duvidoso ou presente), os entrevistadores foram orientados a definirem os casos como de diagnóstico ausente ou presente, melhor refletindo a realidade clínica e de pesquisa. Portanto, os diagnósticos gerados pelo KSADS-E foram computados de forma dicotômica (presente ou ausente) nesse estudo;

b) sugestão de que a concordância entre observadores poderia ser melhor calculada a partir do uso do coeficiente de correlação intraclasse. O coeficiente de correlação intraclasse (CCI) tem a sua concepção teórica baseada em pressupostos similares ao da análise de variança (ANOVA), permitindo, por outro lado, que a relação entre dados sem total independência possa ser avaliada. ${ }^{2}$ Como exemplificado pelas colegas, alguns autores têm sugerido a possibilidade de que o CCI possa também ser utilizado para variáveis binárias (dicotômicas).

Existiria alguma vantagem na utilização do CCI nesse estu- do, ao invés da estratégia adotada? Para responder a essa pergunta, fizemos duas simulações hipotéticas, cada uma com 20 casos avaliados por quatro juizes que poderiam definir o diagnóstico para cada caso de forma dicotômica (presente ou ausente). Na primeira, utilizamos dados que nos fornecessem Kappas 2X2 na faixa considerada de concordância moderada a boa. ${ }^{3} \mathrm{Na}$ segunda simulação, utilizamos apenas dados que nos fornecessem Kappas 2X2 na faixa considerada de concordância excelente (dados disponíveis mediante solicitação).

Considerando os dados da primeira simulação, seis coeficientes Kappa 2X2 foram obtidos: 0,7; 0,8; 0,8;0,9; 0,5; 0,6. A média aritmética desses coeficientes resulta em um Kappa =0,72. Aplicando o CCI, no banco de dados dessa simulação, considerando um modelo do tipo "two-way mixed effect model" (onde existe um efeito randômico para os escores de cada indivíduo, mas não para os escores dos juizes) e determinando uma definição absoluta de concordância, obteríamos um ICC $=0,73 ; \mathrm{p}<0,001$ (considerando um valor de teste $=0$, ou seja, o escore indica que a chance de que a concordância medida seja diferente de zero - refletindo ausência total de concordância - é estatisticamente significativa).

Na segunda simulação, seis coeficientes Kappa 2X2 foram obtidos: 0,$9 ; 0,8 ; 0,9 ; 1,0 ; 0,9 ; 0,9$. A média aritmética desses coeficientes resulta em um Kappa $=0,90$. Novamente, considerando um modelo do tipo "two-way mixed effect model" e determinando uma definição absoluta de concordância, obteríamos um ICC $=0,90 ; p<0,001$. Aliás, essa similaridade de resultados não é surpreendente, pois os próprios autores citados pelas colegas (Ridout et al, 1999) reconhecem que um dos métodos mais antigos de estimar o coeficiente intraclasse é simplesmente o cálculo do coeficiente de correlação de Pearson para todos os pares possíveis de observação que possam ser construídos dentro dos grupos (página 139). Portanto, até que as colegas demonstrem qualquer vantagem na análise proposta, parece desnecessário fazer qualquer re-análise dos dados apresentados nesse estudo.

Finalmente, para leitores tão zelosos, vale ressaltar que todas as comparações dois a dois realizadas produziram coeficientes Kappa considerados de bons a excelentes, pelos critérios de Landis \& $\operatorname{Kock}^{3}$ (dados não apresentados, mas disponíveis mediante solicitação).

\section{Guilherme Polanczyk, Luis Augusto Rohde, Mariana Eizirik, Victor Aranovich, Daniel Denardin, Tatiana L Silva e Thiago G Pianca Programa de Déficit de Atenção/ Hiperatividade (PRODAH) \\ Serviço de Psiquiatria da Infância e Adolescência Hospital de Clínicas de Porto Alegre Universidade Federal do Rio Grande do Sul}

\section{Referências}

1. Polanzyk G, Eizirik M, Aranovich V, Denardin D, Silva TL, Conceição TV et al. Interrater reliability for the schedule for affective disord ers and schizophrenia, epidemiological version for school age children (K-SADSE). Rev Bras Psiquiat 2003;25(2):87-90.

2. Zar J. Biostatistical analysis. $4^{\text {th }}$ ed. New Jersey: Prentice Hall; 1999.

3. Landis J, Koch G. The measurement of observer agreement for categorical data. Biometrics 1977;33:159-74 